\title{
Experimental measurement on movement of spiral-type capsule endoscope
}

\author{
This article was published in the following Dove Press journal: \\ Medical Devices: Evidence and Research \\ 18 January 2016 \\ Number of times this article has been viewed
}

\section{Wanan Yang' \\ Houde Dai ${ }^{2}$ \\ Yong $\mathrm{He}^{1}$ \\ Fengqing Qin'}

'School of Computer and Information Engineering, Yibin University, Yibin, Sichuan, People's Republic of China; ${ }^{2}$ Quanzhou Institute of Equipment Manufacturing, Fujian Institute of Research on the Structure of Matter, Chinese Academy of Sciences,

Quanzhou, People's Republic of China

\begin{abstract}
Wireless capsule endoscope achieved great success, however, the maneuvering of wireless capsule endoscope is challenging at present. A magnetic driving instrument, including two bar magnets, a stepper motor, a motor driver, a motor controller, and a power supplier, was developed to generate rotational magnetic fields. Permanent magnet ring, magnetized as $\mathrm{S}$ and $\mathrm{N}$ poles radially and mounted spiral structure on the surface, acted as a capsule. The maximum torque passing to the capsule, rotational synchronization of capsule and motor, and the translational speed of capsule, were measured in ex vivo porcine large intestine. The experimental results illustrate that the rotational movement of the spiral-type capsule in the intestine is feasible and the cost of the magnetic driving equipment is low. As a result, the solution is promising in the future controllability.
\end{abstract}

Keywords: wireless capsule endoscope, magnet ring, magnetic driving, spiral structure, torque

\section{Introduction}

Wireless capsule endoscope (WCE) was invented at the beginning of this century. ${ }^{1}$ From the day on which it was applied in a clinical test, a great potential was unfolded before our eyes in the diagnosis of small intestinal diseases thanks to its comfortable, painless, and less-invasive exploration of gastrointestinal (GI) tract with a swallowable miniaturized on-board camera. ${ }^{1-6}$ Following the invention, several capsule endoscopes become commercialized, which opens a new era of small intestine inspection. ${ }^{6}$

The main limitation of WCE is its passive and random motion manner, as it is driven by natural peristalsis of the GI tract and gravity. ${ }^{7-10}$ So some interested area could be missed, ${ }^{11}$ in addition, reviewing thousands of pictures is also a burden for a physician. If the controllability for WCE becomes real, the clinical diagnostic efficacy will be greatly improved. More benefits of a controllable WCE might include reduced energy consumption, shortened inspection time and less pictures, and decreased retention risk. ${ }^{12}$

A potential propelling approach is based on an imbedded magnet with the magnetization perpendicular to the capsule's central axis. With the coupling of external revolving magnetic fields, the capsule spins about its axis by magnetic torque. As the spiral thread is attached to the capsule's surface, the rotation can be transformed to translational motion. ${ }^{13,14}$ And the spiral-type capsule has an advantage that the maximum magnetic torque passing to the capsule is proportional to the magnetic field strength, which decreases slower than the gradient magnetic field over the distance. ${ }^{14}$

Some research work about friction and mechanical properties of some parts of GI tract were reported. Wang et al pulled dummy capsules by a string sliding segments of porcine small bowel. ${ }^{15}$ The resistant forces vary from 20 to $100 \mathrm{mN}$ as the capsule 


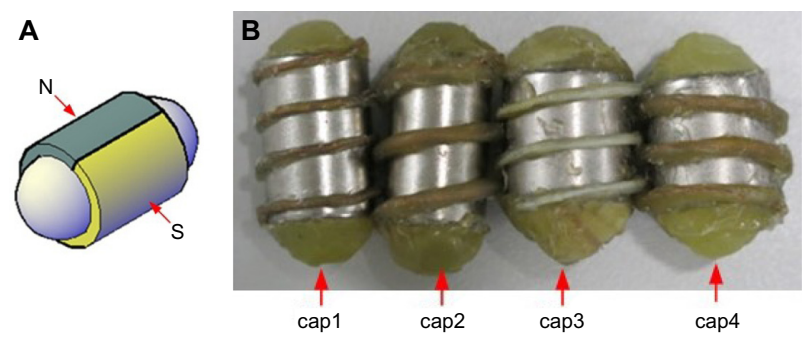

Figure I Poles of magnet ring (A) and fabricated capsules (B) Abbreviations: cap, capsule; N, north; S, south.

diameter is in the range of $8-13 \mathrm{~mm}$ and the moving speed is $0.5 \mathrm{~mm} / \mathrm{s}$. Zhou et al measured torque and friction. ${ }^{14}$ Natali et al measured the resistant force against the propulsion of WCE through the coupling of the external magnet. ${ }^{16}$ Aforementioned work is of benefit to the understanding of the properties of the GI tract. Physical connection to the outside instrument may lead to inaccuracy. In this study, a noncontact measurement approach was designed. The magnetic permeability of the human body is very close to that of vacuum, so human body will not change the distribution of static magnetic field. ${ }^{17}$ We propose that a cylindrical magnet ring radially magnetized as $\mathrm{S}$ (south) and $\mathrm{N}$ (north) poles covers outside the commercial WCE, leaving the dome parts uncovered. The magnet ring is covered by biomedical polymer material, avoiding direct contact with mucus. A spiral structure is mounted on the surface of WCE, converting the rotation into straight motion. The movement of spiral-type WCE rotating inside the GI tract was investigated in a realistic manner. A small magnetic driving instrument (MDI) was specially designed for experiment inspired by Lien et al. ${ }^{18}$ The segments of porcine large intestine were deployed for the ex vivo experiments. Four capsule dummies were fabricated for propulsion efficiency and moving speed exploring, and it can be used as reference when developing a real WCE.

\section{Basic experimental setup}

Four spiral-type dummy capsules were fabricated. Each capsule, the hollow cylindrical permanent magnet (magnet ring), radially magnetized as $\mathrm{S}$ and $\mathrm{N}$ poles (Figure $1 \mathrm{~A}$ ), was filled with ethylene-vinyl acetate copolymer at the both ends of the magnet ring to form domes, simulating the shape of

Table I Parameters of magnet rings

\begin{tabular}{lllll}
\hline Identifier & $\begin{array}{l}\text { Length } \\
(\mathbf{m m})\end{array}$ & $\begin{array}{l}\text { Outer } \\
\text { diameter } \\
(\mathbf{m m})\end{array}$ & $\begin{array}{l}\text { Inner } \\
\text { diameter } \\
(\mathbf{m m})\end{array}$ & $\begin{array}{l}\text { Maximum magnetic } \\
\text { flux intensity of two } \\
\text { poles }(\mathbf{m} \mathbf{)})\end{array}$ \\
\hline ring I & 18 & 12 & 10 & 247 \\
ring2 & 15 & 15 & 12 & 340 \\
\hline
\end{tabular}

Table 2 Parameters of dummy capsules

\begin{tabular}{llll}
\hline Identifier & $\begin{array}{l}\text { Total length } \\
(\mathbf{m m})\end{array}$ & $\begin{array}{l}\text { Diameter of } \\
\text { spiral line } \mathbf{( m m})\end{array}$ & $\begin{array}{l}\text { Magnet ring } \\
\text { identifier }\end{array}$ \\
\hline capl & 28 & $\mathrm{I}$ & ringl \\
cap2 & 28 & 2 & ringl \\
cap3 & 26 & 1 & ring2 \\
cap4 & 26 & 2 & ring2 \\
\hline
\end{tabular}

Abbreviation: cap, capsule.

commercial WCE. NdFeB has a stronger magnetic field in the ordinary environment, so we chose $\mathrm{NdFeB}$ (grade: N38) as a raw material for magnet rings. The parameters of the magnet ring are listed in Table 1, and the magnetic flux intensities were measured using Tesla meter (HT20; Shanghai Hengtong Cidian Technology Co, Ltd, Shanghai, People's Republic of China). To ensure authenticity, the dimensions of magnet rings are made to approximate those of commercial WCEs on the market. A segment of copper wire coated with ethylene-vinyl acetate copolymer was wound on the surface of each magnet ring and acted as spiral structure. The winding part was only within the cylindrical magnet surface, as shown in Figure 1B. Two diameters of spiral line were tentatively chosen, and the length of each dummy capsule is described in Table 2 .

The small MDI included a stepper motor, a motor driver, a controller, a power supplier, and two same bar permanent magnets (length: $5 \mathrm{~cm}$; width: $0.8 \mathrm{~cm}$; height: $0.6 \mathrm{~cm}$ ), which were also made of $\mathrm{NdFeB}$ (grade: N38; maximum magnetic flux intensity of poles: $595 \mathrm{mT}$ ). Two bar magnets were fixed on the axis of the motor with opposite pole close together, and the stepper motor was used to rotate the bar magnets to generate rotational magnetic fields. The rotational speed of the stepper motor was adjusted conveniently by changing the parameters of imbedded program. In addition, the rotational speed of stepper motor was accurately measured using

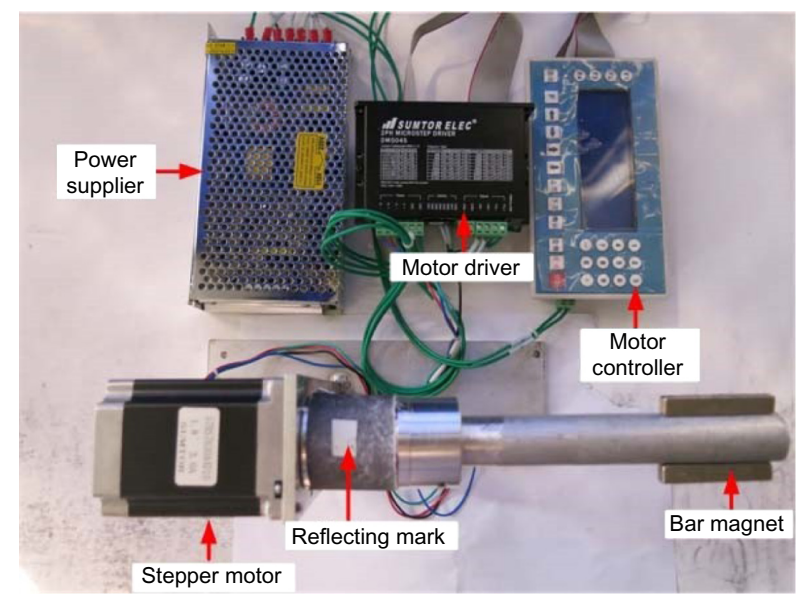

Figure 2 General view of magnetic driving instrument. 


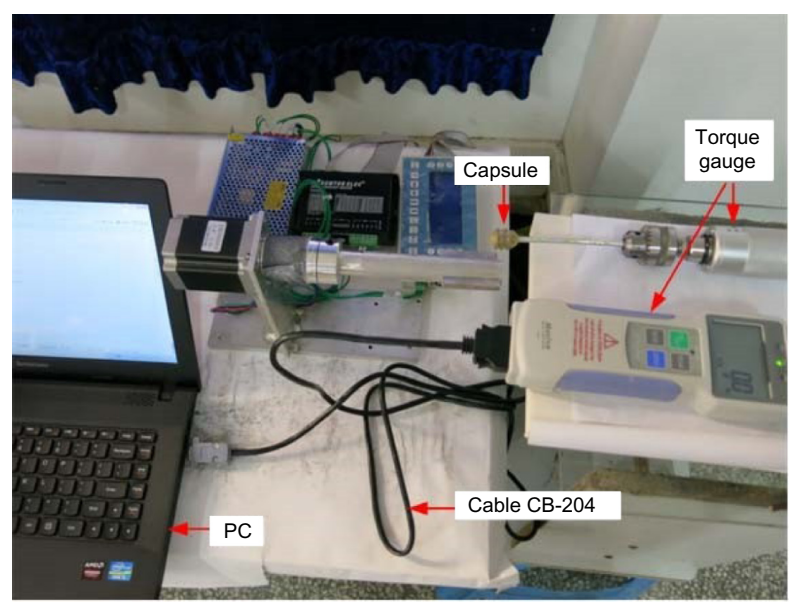

Figure 3 The torque measurement setup.

Abbreviations: PC, personal computer; CB-204, cable-204.

an infrared rotation meter (DT-2234B; LUTRON, Taibei, Taiwan). A piece of reflecting mark was stuck on the surface of the connector to implement noncontact measurement. The general view of MDI is illustrated in Figure 2.

The experiments were carried out in the ordinary lab at room temperature of $25^{\circ} \mathrm{C}$. To guarantee the freshness of the intestine, the intestines of pig were bought in the early morning from the slaughterhouse. It was approximately 1 hour after the pork intestine was pulled out that the experiments began. To keep the characteristic of the live specimen, the normal saline was sprayed on the surface of the intestine every 20 minutes. Translational time measurements were completed within 2 hours.

\section{Experimental measurement and result \\ Torque measurement}

The torque generated by the rotational magnetic fields will pass to the magnetic ring. To find what torque can rotate the capsule, the rigid rod was fastened to one end of the capsule, and the other end of the rod was connected to the handy torque gauge (HTG2-5N; IMADA, Toyohashi, Japan), which was

Table 3 Torque measurements

\begin{tabular}{ll}
\hline Distance $(\mathbf{m m})$ & Torque $(\mathbf{g f} \cdot \mathbf{m})$ \\
\hline 15 & 4.75 \\
20 & 3.20 \\
25 & 2.07 \\
30 & 0.93 \\
35 & 0.33 \\
40 & 0.14 \\
45 & 0.09 \\
50 & 0.001 \\
\hline
\end{tabular}

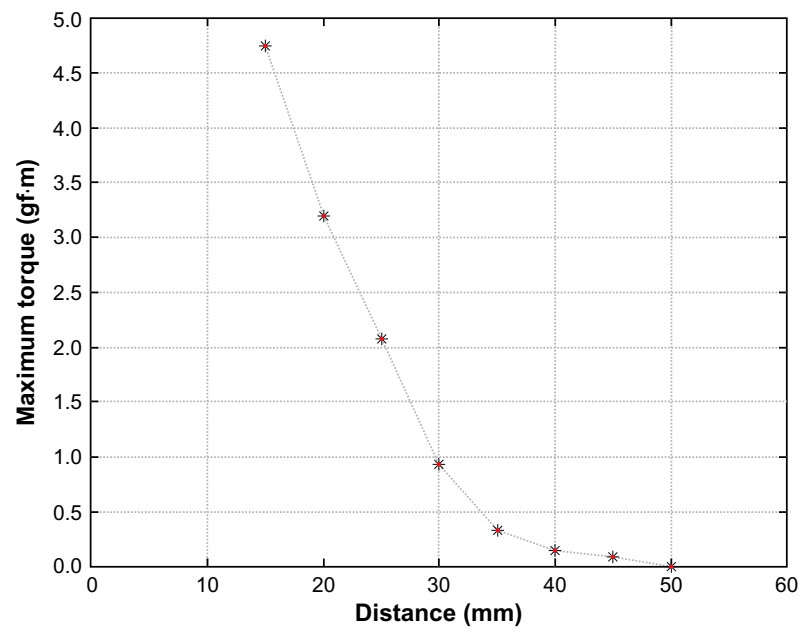

Figure 4 The torque measurement.

able to display the real-time data in the indicator. Through RS232C cable CB-204 and application software provided by IMADA, the real-time data were transferred to PC for storing and further processing. The torque measurement setup is shown in Figure 3.

Suppose the parameters of magnet ring are as follows: the length $l$, inner radius $r_{\mathrm{i}}$, outer radius $r_{\mathrm{o}}$, and magnetization $M_{0}$ (magnetic dipole moment per unit volume). Then the magnetic dipole moment $\mathbf{m}$ of the magnet ring can be formulated as

$$
\mathbf{m}=\iiint_{v} M_{0} d v=M_{0} \pi\left(r_{\mathrm{o}}^{2}-r_{\mathrm{i}}^{2}\right) l
$$

The magnetic torque $\mathrm{T}$ produced on a dipole moment $\mathbf{m}$ by the applied magnetic flux intensity $\mathbf{B}$ causes $\mathbf{m}$ to align with $\mathbf{B}$ and is expressed by

$$
\mathrm{T}=\mathbf{m} \times \mathbf{B}
$$

Because the magnetic fields produced by both the magnet ring and rotational magnet are not uniform, the magnetic torques vary from 0 to maximum. The torques change slightly with different capsules, cap1 is used to measure the torques

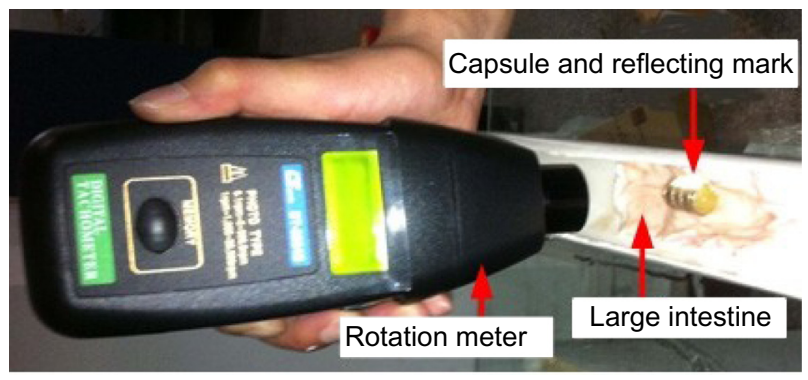

Figure 5 Measurement setup of rotation synchronization of capsule and stepper motor. 

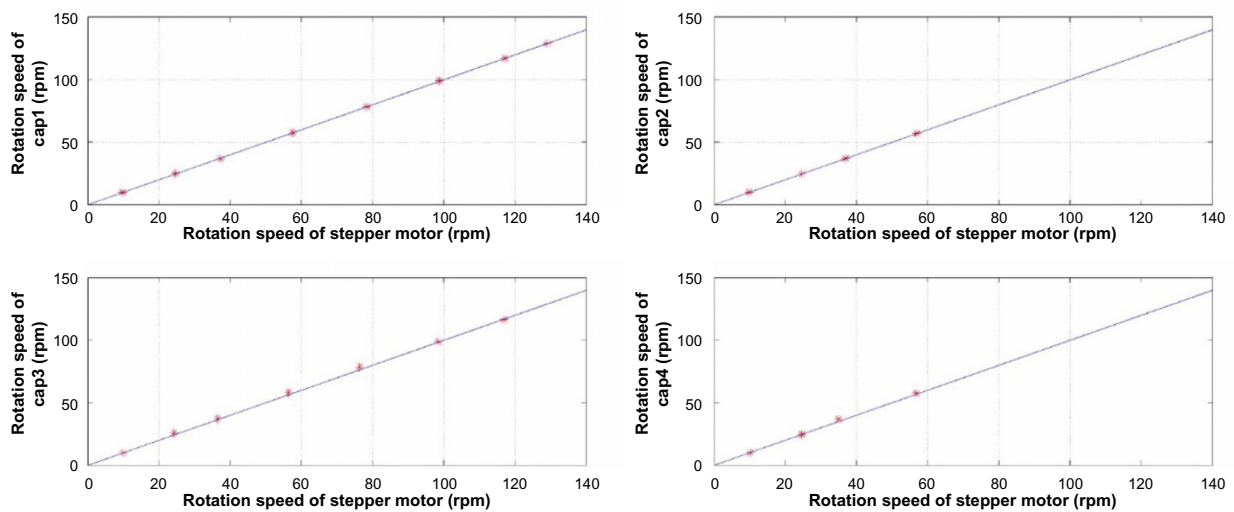

Figure 6 The measurement of rotation synchronization of capsule and stepper motor. Abbreviations: rpm, revolutions per minute; cap, capsule.

to understand the general magnitude and variation trend. The distance between the center of the capsule and the surface of rotational magnet was fixed. When the motor rotates one circle, the maximum value in one cycle was automatically recorded by torque meter. From Table 3, we can infer what torque can rotate the capsule when the distance is determined. Figure 4 illustrates that the torque decreases quickly with the increasing distance, which is reliable due to quick decrease of the magnetic flux intensity $\mathbf{B}$ over distance.

\section{Rotational synchronization measurement}

Rotational synchronization of capsule and stepper motor reflects the efficiency of rotation propelling. To implement measurement of rotational speed of the capsule, the reflecting mark was attached on the surface of the capsule. A short segment of porcine large intestine was put in the half pipe, and the capsule was put on the surface of arc-shape large intestine. When the capsule rotated, the infrared of the rotation meter pointed at the reflecting mark, and then the rotation speed of the capsule was automatically recorded. The measurement view is shown in Figure 5. The rotational speed of stepper motor was also measured using the similar approach. All dummy capsules were used to investigate the synchronization in the experiment.

The rotational speeds of stepper motor were recorded as follows: 9.7, 24.6, 37.3, 57.6, 78.3, 98.7, 117.1, and $128.9 \mathrm{rpm}$. Four dummy capsules were propelled by these rotational speeds separately. Due to trembling of cap2 and cap4 when the rotational speed of motor exceeded $70 \mathrm{rpm}$, the rotational speed of capsule cannot be correctly recorded. As the diameters of a spiral line of both cap2 and cap4 are two times larger than cap1 and cap3, we cautiously infer that the diameter of spiral line is larger. The trembling is more intensive.

Figure 6 illustrates that the rotation speeds of cap 1 and cap3 highly match the rotational speeds of the stepper motor. The rotational speeds of cap2, cap4, and stepper motor are

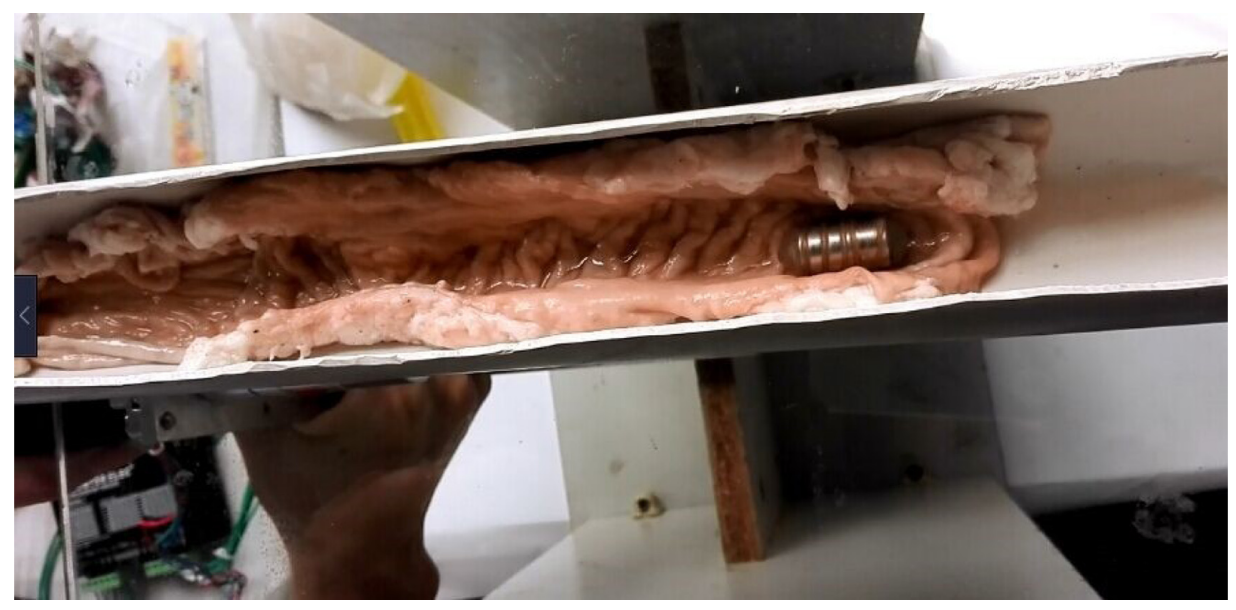

Figure 7 Measurement setup of translational motion time. 
Table 4 Translational motion time

\begin{tabular}{llllllllllll}
\hline $\begin{array}{l}\text { Rotational } \\
\text { speed of } \\
\text { motor (rpm) }\end{array}$ & 216 & 177 & 150 & 129 & 117 & 99 & 78 & 57 & 36 & 24 & 9 \\
$\begin{array}{l}\text { Minimum time } \\
\text { capl seconds) }\end{array}$ & 32 & 36 & 33 & 30 & 37 & 39 & 35 & 42 & 58 & 88 & $*$ \\
capl & 33 & 35 & 41 & 46 & 45 & 52 & 72 & 70 & 79 & 132 & $*$ \\
cap2 & 36 & 51 & 31 & 42 & 56 & 41 & 68 & 65 & 83 & 85 & $*$ \\
cap3 & 27 & 36 & 40 & 34 & 43 & 45 & 43 & 49 & 58 & 80 & $*$ \\
cap4 & & & & & & & & & & &
\end{tabular}

Note: *Indicates that the capsule hardly moved forward at the rotational speed of 9 rpm.

Abbreviations: rpm, revolutions per minute; cap, capsule.

also highly matched, though the rotational speed of capsule remains unknown when the rotational speeds of stepper motor exceed $60 \mathrm{rpm}$.

\section{Translational movement}

Due to no reference to the length of porcine large intestine, after several times of trials, we found that $40 \mathrm{~cm}$ intestine is suitable for measurement. The large intestine with $40 \mathrm{~cm}$ was cut open and put into the half pipe, keeping arc-shaped, and the capsule was placed at the end of the intestine, as illustrated in Figure 7. For each capsule, the stepper motor's rotational speed varied from 9 to $216 \mathrm{rpm}$. When rotating, the stepper motor moved forward under the half pipe at the speed of $0.5-2 \mathrm{~cm} / \mathrm{s}$, driving the capsule to rotationally move forward. The three measurements of minimum time were recorded, and the average value was used to calculate the translational velocity of the capsule.

The measured data are listed in Table 4. The asterisk "*” means that the capsule hardly moves forward at the rotational speed of 9 rpm. As shown in Figure 8, the translational speed of cap1 almost increases linearly with increasing rotational speed when the rotational speed of the stepper motor is less than $80 \mathrm{rpm}$. When the speed of the stepper motor exceeds $80 \mathrm{rpm}$, the translational speed of cap1 keeps stable. The translational speeds of cap2, cap3, and cap4 increase slowly when the stepper motor's rotational speeds are less than $80 \mathrm{rpm}$, and we observed that their stabilities are not so good as cap1. The capsule trembled when the rotational speed of stepper motor reached approximately $150 \mathrm{rpm}$. From the view of propelling efficiency, it is delightful because the trembling can help the capsule to overcome the resistance produced by the fold of the intestine. We preliminarily conclude that cap 1 is better than others from the aspects of the dimension and helical line.

\section{Discussion}

The spiral-type capsule has the ability to rotationally move forward at the speed of over $1 \mathrm{~cm} / \mathrm{s}$. The total intestine of an adult is approximately $10 \mathrm{~m}$. If the capsule advances at this speed, less than 20 minutes will be cost when the capsule passes through the total intestine. Thus, the inspection time will be shortened greatly.
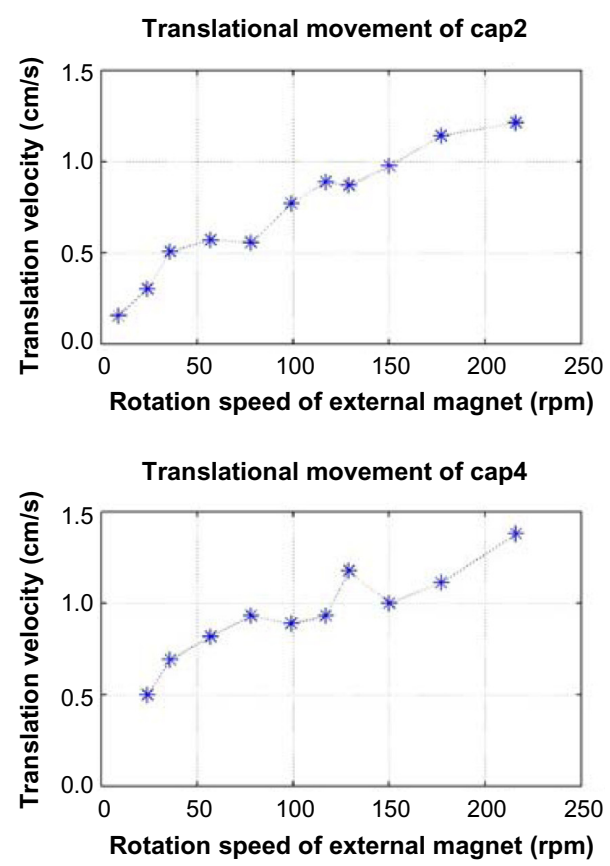

Figure 8 Translational velocity of capsules under different rotation speed. Abbreviations: rpm, revolutions per minute; cap, capsule. 
When the capsule rotationally moved forward in the experiment, the distance between the external magnet and capsule was kept approximately $3.5 \mathrm{~cm}$, which is challenging for the obese people. Due to fast decay of the magnetic field strength of permanent magnet over distance, the torque generated by the permanent magnet quickly decreases, so extending the effective distance of MDI is essential.

Furthermore, the peristalsis of the living intestine may resist against the movement of the capsule, even push back the capsule, we will further investigate the motion of the capsule after approval. Thus, we need experimental investigations to determine the appropriate lead angle and the diameter of the spiral line for capsule to move in the living intestine.

The mucus, water, and debris in the intestine may protect the wall of intestine in some degree when the capsule rotates. However, we found that the rotation of spiral structure will push forward some mucus, water, and debris. Further investigations are needed in the future to know whether the removal of mucus has bad influences on living body physiologically.

\section{Conclusion}

The straight movement of the spiral-type capsule is experimentally demonstrated in the viscoelastic intestine. The rotation of spiral-type capsule is converted to translational movement effectively, and the translational speed of capsule can exceed $1 \mathrm{~cm} / \mathrm{s}$. The rotations of the capsule and the stepper motor are highly synchronized. The validity of this driving approach in the winding intestine and optimal parameters of spiral structure will be investigated further.

\section{Acknowledgments}

This project is supported by the National Natural Science Foundation of China (grant numbers 61202196, 61273332, 61202195), Scientific Research Fund of Sichuan Provincial Education Department (grant number 12ZA200), Yibin Scientific and Technological Project (grant number 2013ZSF010), and also supported by Doctoral Fund of Yibin University (grant number 2011B06).

\section{Disclosure}

The authors report no conflicts of interest in this work.

Medical Devices: Evidence and Research

\section{Publish your work in this journal}

Medical Devices: Evidence and Research is an international, peerreviewed, open access journal that focuses on the evidence, technology, research, and expert opinion supporting the use and application of medical devices in the diagnosis, treatment and management of clinical conditions and physiological processes. The identification of novel

\section{References}

1. Iddan G, Meron G, Glukhovsky A, Swain P. Wireless capsule endoscopy. Nature. 2000;405:417.

2. Meng MQ-H, Mei T, Pu J, Hu C, Wang X, Chan Y. Wireless robotic capsule endoscopy: state-of-the-art and challenges. In: Proceedings of the 5th World Congress on Intelligent Control and Automation; June 15-19, 2004; Hangzhou, People's Republic of China.

3. Moglia A, Menciassi A, Dario P, Cuschieri A. Capsule endoscopy: progress update and challenges ahead. Nat Rev Gastroenterol Hepatol. 2009;6:353-361.

4. Rasouli M, Kencana AP, Van An Huynh, Kiat Ting E, Lai JCY, Phee LSJ. Wireless capsule endoscopes for enhanced diagnostic inspection of gastrointestinal tract. In: 2010 IEEE Conference on Robotics Automation and Mechatronics; June 28-30, 2010; Singapore.

5. Waghmare PV, Panchal CV, Poul BN. Swallowable wireless capsular endoscopy: a novel breakthrough in the biomedical industry and future progress. Int J Pharm Sci Res. 2013;4:4133-4144.

6. Fisher LR, Hasler WL. New vision in video capsule endoscopy: current status and future directions. Nat Rev Gastroenterol Hepatol. 2012;97:392-405.

7. Carpi F. Magnetic capsule endoscopy: the future is around the corner. Expert Rev Med Devices. 2010;7:161-164.

8. Carpi F, Shaheed H. Grand challenges in magnetic capsule endoscopy. Expert Rev Med Devices. 2013;10:433-436.

9. Carpi F, Galbiati S, Carpi A. Controlled navigation of endoscopic capsules: concept and preliminary experimental investigations. IEEE Trans Biomed Eng. 2007;54:2028-2036.

10. Keller H, Juloski A, Kawano H, et al. Method for navigation and control of a magnetically guided capsule endoscope in the human stomach. In: The Fourth IEEE RAS/EMBS International Conference on Biomedical Robotics and Biomechatronics; June 24-27, 2012; Rome, Italy.

11. Liao Z, Duan X, Xin L, et al. Feasibility and safety of magnetic-controlled capsule endoscopy system in examination of human stomach: a pilot study in healthy volunteers. J Interv Gastroenterol. 2012;2:155-160.

12. Quirini M, Menciassi A, Scapellato S, et al. Feasibility proof of a legged locomotion capsule for the GI tract. Gastrointest Endosc. 2008;67: 1153-1158.

13. Sendoh M, Yamazaki A, Chiba A, Soma M, Ishiyama K, Arai KI. Spiral type magnetic micro actuators for medical applications. In: Proceedings of the 2004 International Symposium on Micro-Nanomechatronics and Human Science. Piscataway, NJ: IEEE; 2004:319-324.

14. Zhou H, Alici G, Than TD, Li W. Modeling and experimental investigation of rotational resistance of a spiral-type robotic capsule inside a real intestine. IEEE ASME Trans Mechatron. 2013;18:1555-1561.

15. Wang X, Meng MQ-H. An experimental study of resistant properties of the small intestine for an active capsule endoscope. Proc Inst Mech Eng H. 2010;224:107-118.

16. Natali CD, Beccani M, Obstein KL, Valdastri P. A wireless platform for in vivo measurement of resistance properties of the gastrointestinal tract. Physiol Meas. 2014;35:1197-1214.

17. Hu C. Localization and Orientation System for Robotic Wireless Capsule Endoscope [dissertation]. Edmonton, AB: University of Alberta; 2006:16.

18. Lien G-S, Liu C-W, Jiang J-A, Chuang C-L, Teng M-T. Magnetic control system targeted for capsule endoscopic operations in the stomach design, fabrication, and in vitro and ex vivo evaluations. IEEE Trans Biomed Eng. 2012;59(7):2068-2079.

\section{Dovepress}

devices and optimal use of existing devices which will lead to improved clinical outcomes and more effective patient management and safety is a key feature. The manuscript management system is completely online and includes a quick and fair peer-review system. Visit http://www. dovepress.com/testimonials.php to read real quotes from authors. 